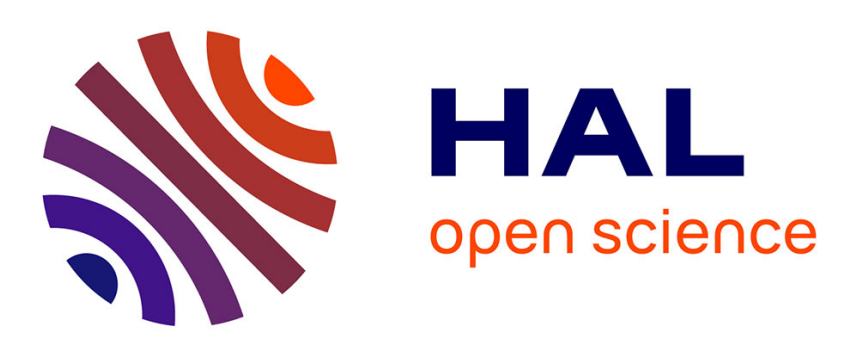

\title{
RICHIE: A Step-by-step Navigation Widget to Enhance Broad Hierarchy Exploration on Handheld Tactile Devices
}

\author{
Alexandre Kabil, Sébastien Kubicki
}

\section{- To cite this version:}

Alexandre Kabil, Sébastien Kubicki. RICHIE: A Step-by-step Navigation Widget to Enhance Broad Hierarchy Exploration on Handheld Tactile Devices. 17th International Conference, HCI International 2015, Aug 2015, Los Angeles, United States. pp.196-207, 10.1007/978-3-319-20916-6_19 . hal-01264407

\section{HAL Id: hal-01264407 https://hal.science/hal-01264407}

Submitted on 29 Jan 2016

HAL is a multi-disciplinary open access archive for the deposit and dissemination of scientific research documents, whether they are published or not. The documents may come from teaching and research institutions in France or abroad, or from public or private research centers.
L'archive ouverte pluridisciplinaire HAL, est destinée au dépôt et à la diffusion de documents scientifiques de niveau recherche, publiés ou non, émanant des établissements d'enseignement et de recherche français ou étrangers, des laboratoires publics ou privés. 


\title{
RICHIE: a step-by-step navigation widget to enhance broad hierarchy exploration on handheld tactile devices
}

\author{
Alexandre Kabil and Sébastien Kubicki \\ ENI Brest, Lab-STICC UMR6285, 29200 Brest, France \\ $\{$ kabil, kubicki\}@enib.fr
}

\begin{abstract}
Exploring large hierarchies is still a challenging task, especially for handheld tactile devices, due to the lack of visualization space and finger's occlusion. In this paper, we propose the RICHIE (Radial InCremental HIerarchy Exploration) tool, a new radial widget that allows step-by-step navigation through large hierarchies. We designed it to fit handheld tactile requirements such as target reaching and space optimization. Depth exploration is made by shifting two levels of hierarchy at the same time, for reducing the screen occupation. This widget was implemented in order to adapt a Command and Control (C2) system to mobile tactile devices, as these systems require the on-screen presence of an important unit's hierarchy (the ORder of BATtle). Nevertheless, we are convinced that RICHIE could be used on several systems that require hierarchical data exploration, such as phylogenetic trees or file browsing.
\end{abstract}

Keywords: multi-touch, information visualization, hierarchy

\section{Introduction}

Various existing interaction and visualization techniques for large datasets aim at displaying all linked items, using node-link or space-filling approaches [1]. Enhanced node-link approaches give the opportunity to visualize and select elements from various datasets, whereas radial space-filling ones are centered on hierarchical data [2]. These approaches have drawbacks when used on mobile devices, especially when datasets are substantial. To overcome these limitations, we propose RICHIE, a Radial InCremental Hierarchy Exploration widget composed of two concentric quadrant shapes, which are placed at a bottom corner of the screen, facilitating thumb usability. The first arc contains a first level selected item and its siblings, whereas the second arc contains its children.

Our approach is inspired by ControlTree [3], which defines three zones for a selected item: a sibling zone, a children zone and a parent zone. However, for minimizing occupation space, we display only few items at once from sibling and children zones; circular dragging gesture on quadrants allow navigation through hidden items. Moreover, depth exploration is made selecting an item on the 
second quadrant and by shifting itself and its siblings to the first quadrant (forward navigation) or by shifting items from the first quadrant to the second one, allowing items from the parent zone to appear on the first arc (backward navigation).

We choose this specific design because we do not want our widget to occupy the whole screen area, like Radial Edgeless Tree [4] or Radial Space Filling [5] methods, as we implemented it in a C2 (Command and Control) system [6], in which users interact mainly on a map but also need to control a large number of hierarchical units (Figure 1).

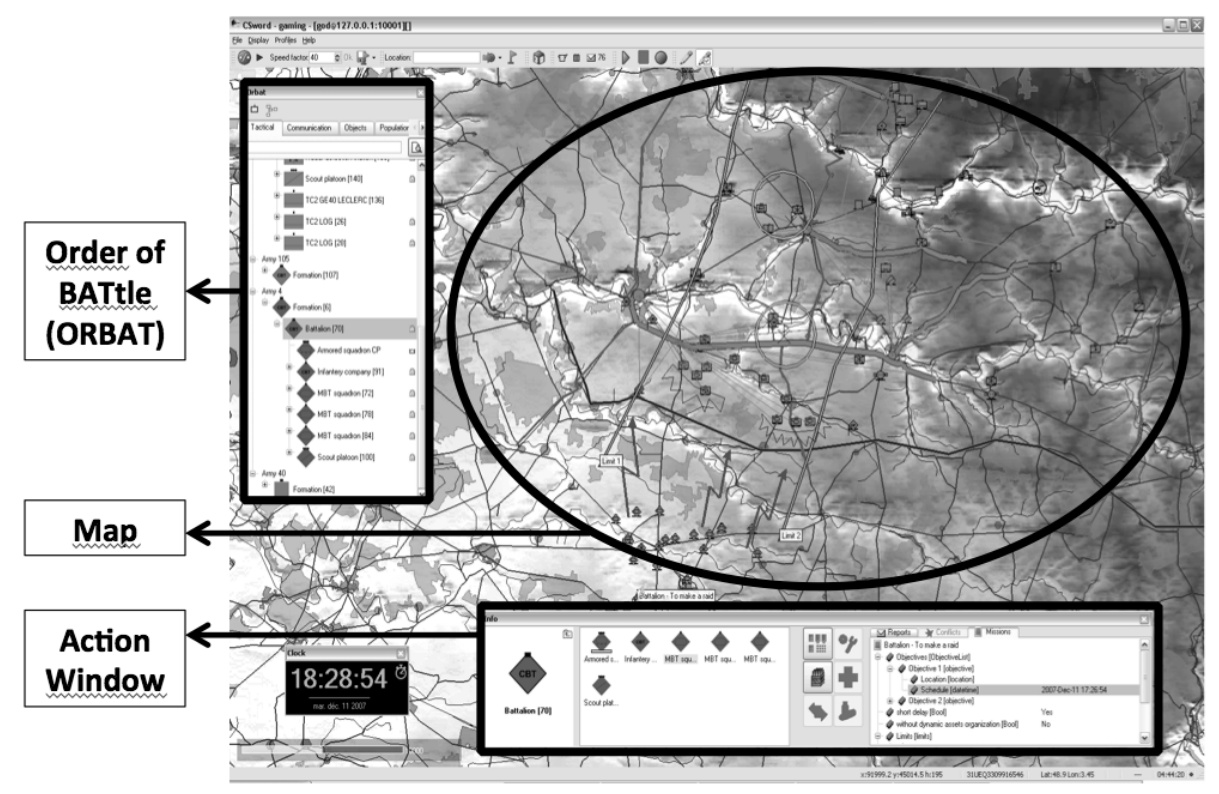

Fig. 1. View of the existing C2 System.

Finally, we think that our widget can be used in different domains, such as files browsing or phylogenetic trees exploration, which requires exploration of large hierarchical datasets.

This paper is organized as follows. First, we will give a review of the current hierarchy exploration techniques and especially those that can be used on tactile devices. Then we will present our RICHIE widget, designed to overcome broad hierarchy navigation issues on mobile tactile devices. The next section will describe in details the functioning of the widget, and the different interaction techniques that we are developing in order to evaluate them from a user's point of view. Finally, some considerations on the usability of our widget in different domains will be exposed. 


\section{Related Work}

There exists numerous hierarchy visualization and navigation techniques, but we can classify them into two categories [1,4]: (1) node-link, connection or explicit visualization techniques [4] aim at representing relations between connected data with semantic links whereas (2) space-filling, enclosure or implicit techniques [7] use positions and sizes of nodes to convey hierarchical meaning.

\section{$2.1 \quad$ Node-edge approaches}

Hao et al. [4] show that major issues for node-link displaying approaches are the location and the connection of the nodes. Visualization's optimization of graphs and especially hierarchical ones have been exhaustively researched, and approaches like H-Tree or Radial trees [8] (Figure 2) are convenient for simple desktop usages. But, when users want to interact with graphs, they need adapted interaction techniques. ControlTree [3], for instance, helps user by displaying siblings and children of selected item in specific spaces (Figure 3). However, these visualization and interaction techniques are not well designed for tactile devices, due to the lack of cursor and fingers occlusion, which limit precise interaction. That is why there is a growing need in Information Visualization domain for novel interaction techniques that enables multi-touch graph interaction [9].

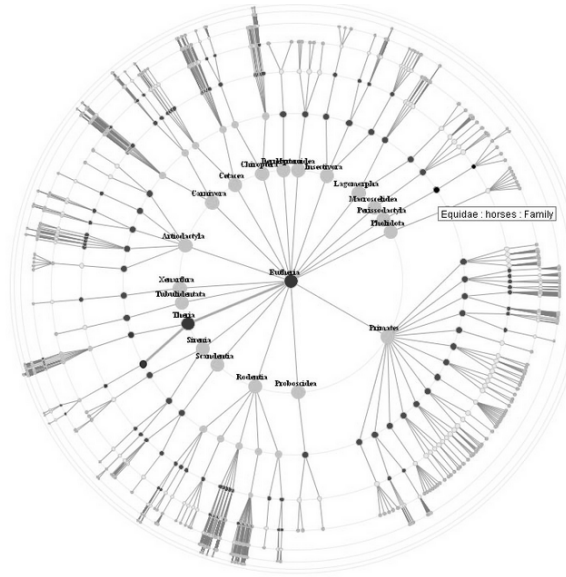

Fig. 2. Radial Tree Visualization [8]

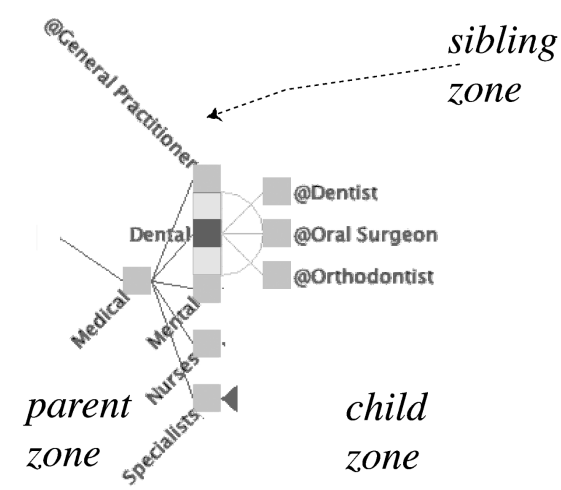

Fig. 3. ControlTree zones [3]

More recently, Holzinger et al. [10] made a multi-touch graph-based interaction review that highlights the techniques used and the challenges new devices offers for information visualization. Yet, for hierarchical graphs, where links between nodes are quite implicit (parents-children or siblings semantic relationships), the space-filling approach seems more adapted to mobile devices, even 
if some mixed techniques such as SpaceTree [11] or EnCon [12] tried to take advantage of both approaches by adapting a graph to a specified size.

\section{$2.2 \quad$ Space-filling approaches}

Links in enclosure visualization techniques are implicit, and the geometric positions and sizes of nodes reveals the nature of their connections. Treemap [13] is the most simple space-filling approach, but lacks clarity regarding the hierarchy (Figure 4). That is why, radial or concentric techniques were developed [2], such as InterRing [5] (Figure 5). RELT (Radial EdgeLess Tree), developed by Hao et $a l$. is a visualization technique that aims at maximizing the space used by data and providing a clear hierarchy view [4]. These techniques are less convenient when hierarchies are imbalanced (when items have very different number of siblings or children). To curb this issue, Chhetri et al. proposed ERELT (Enhanced RELT) [7] which allows users to perform a drag gesture in order to display hidden items, allowing the visualization of large hierarchies without reducing node sizes (Figure 6).

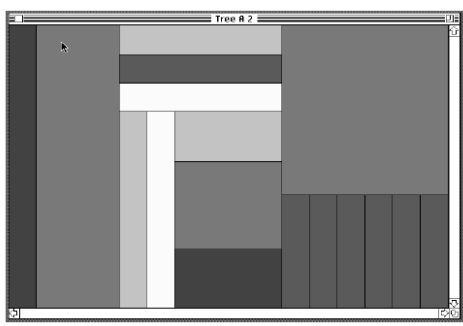

Fig. 4. TreeMap [13]

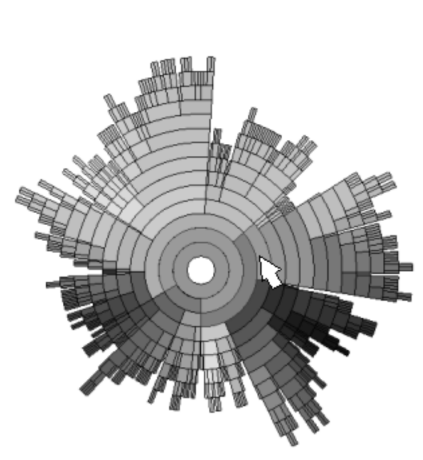

Fig. 5. InterRing [5]

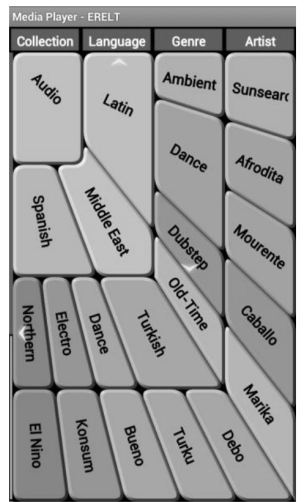

Fig. 6. ERELT [7]

All of approaches made for mobile devices tends to maximize the utilization of screen area, which sometimes is not required, when for example you do not want to lose the view of an application while browsing some data hierarchy . Moreover, the size-changing of items could limit finger interaction, if their number is too important.

In the next section we will introduce RICHIE, a step-by-step widget that tends to curb these issues. 


\section{Design}

We designed the RICHIE technique with the constraints that it should not take whole screen space, items size should be finger-adapted, and the interaction should be easy on mobile tactile devices, such as tablets or smartphones.

\subsection{Concentric shape}

The radial concentric shape was proved efficient for displaying hierarchical data [7]. Moreover, putting the center of widget in a bottom corner of the device facilitates thumb [14] or two-hands interaction [15], due to the grasping position and the physiology of hands [16]. By taking inspiration from the wheel metaphor [14], we decided to consider a quadrant radial design, similar to a corner menu [17]. But, we do not want all items of the same hierarchy level to be visible, so, as ERELT [7], we display only a part of items and user can access hidden ones by dragging them on the active view. This design allows us to make all items the same size (Figure 7), which can fit through tactile guidelines [18], and not to limit the broadness of hierarchy datasets.

Moreover, to facilitate lisibility of hierarchical links between items, selection of a first quadrant's item puts it at $45^{\circ}$, in a highlight zone (Figure 7 ), whereas its children appears on second arc.

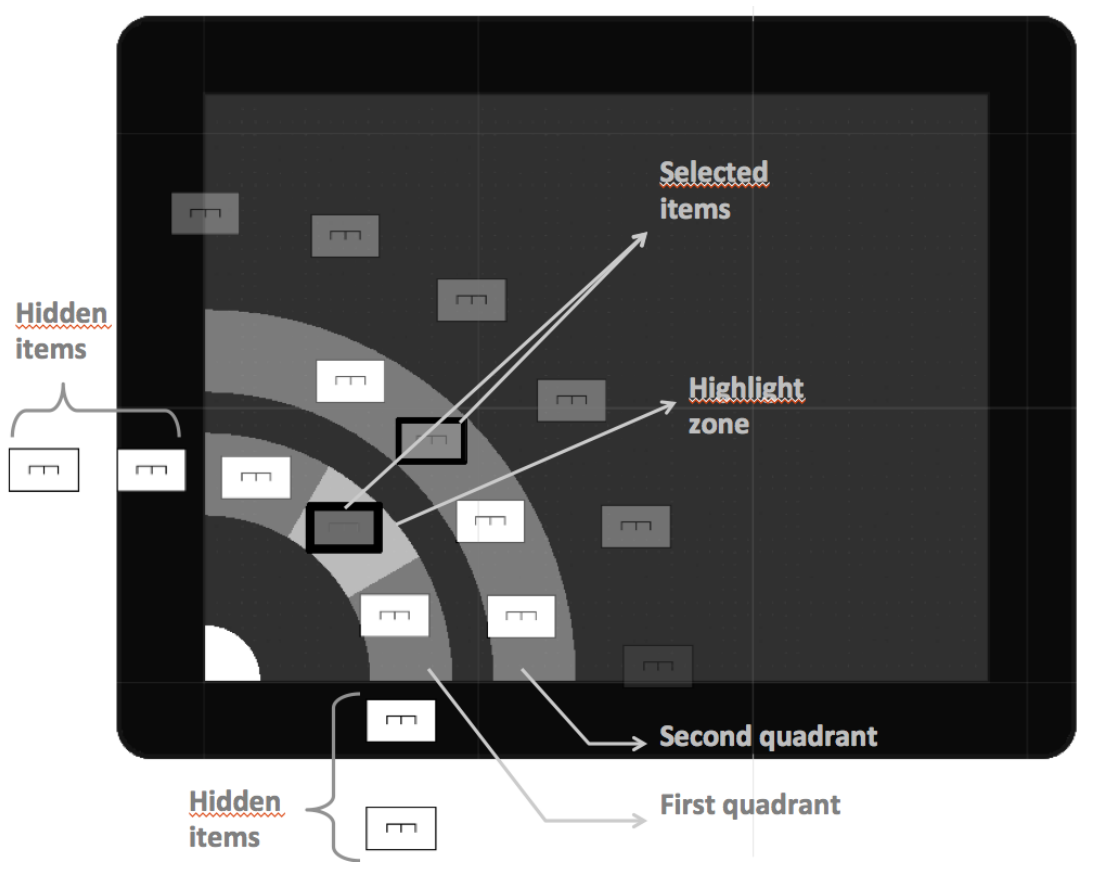

Fig. 7. Prototype view of item's positionning. 


\subsection{Navigating through hierarchy}

We called our widget RICHIE (Radial InCremental HIerarchy Exploration) because we do not want to display the whole hierarchy at the same time. We propose a navigation technique that is different from Moire graph [19], Stackedhalf-pie [20] and wavelet menus [21], because we only want to interact with two levels of hierarchy, for limiting screen occupation (Figure 11). The first quadrant contains selected item and its siblings whereas second one contains selected item s children (Figure 8). We took inspiration from ControlTree [3] zones, namely sibling zone, children zone and parent zone which is hidden in our widget. Forward navigation (Figures 9) is made by dragging second quadrant s selected item to the first quadrant and backward navigation (Figure 10) by dragging first arc 's selected item to the second one. Drag and tap gestures used in this navigation technique are considered direct manipulation gestures, and are proved effective for selection tasks $[22,23]$.
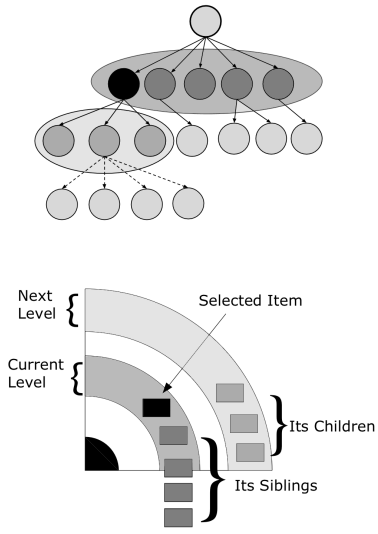

Fig. 8. Selection of a first arc's item
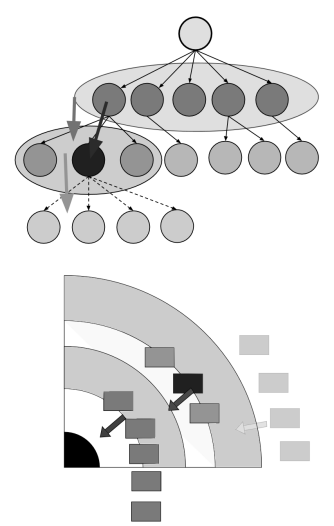

Fig. 9. Forward navigation
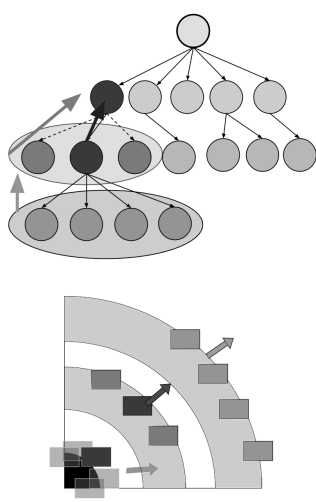

Fig. 10. Backward navigation

Fig. 11. Different states of the widget compared to a hierarchical view

The next section will provide information about development issues and choices.

\section{Implementation and development}

We developed our widget with Unity3D, a real-time game engine. This choice was made because this software allowed us to design the shapes and behaviors we wanted, in a framework that takes into account tactile events and which is more evolutive than sketching or prototyping tools, partly because of its multiplatform capabilities. 


\subsection{Display and interaction with items}

We designed a unique function that allows positioning of same level hierarchy items. Let $U$ being a list of $M$ items and $N$ the number of visible elements we want on our widget's quadrants $(N>1)$. By taking the center of widget, horizontal and vertical axes of device for geometrical frame, position $\overrightarrow{U_{i}}$ of $U[i]$ item will depends on two coordinates and its angle $\theta_{i}$ with abscissa axis, given the radius $R$ of the display circle (Equation 1 ):

$$
\theta_{i}=\frac{i \times \pi}{2(N-1)}+C
$$

Where $C$ is a variable controlled by dragging: dragging to the upper side of the widget increases $\mathrm{C}$ whereas dragging to the bottom side decreases it. Initial positions of $U$ 's items are, for $i \in[0, M-1]$ (Equations 2, 3,4) :

$$
\begin{aligned}
& \text { If } \theta_{i}<0: \quad \overrightarrow{U_{i}} \cdot \vec{x}=R ; \quad \overrightarrow{U_{i}} \cdot \vec{y}=-\theta_{i} \times K \\
& \text { If } \theta_{i}<\frac{\pi}{2}: \quad \overrightarrow{U_{i}} \cdot \vec{x}=R \times \cos \left(\theta_{i}\right) ; \quad \overrightarrow{U_{i}} \cdot \vec{y}=R \times \sin \left(\theta_{i}\right) \\
& \text { Else : } \quad \overrightarrow{U_{i}} \cdot \vec{x}=\left(\frac{\pi}{2}-\theta_{i}\right) \times K ; \quad \overrightarrow{U_{i}} \cdot \vec{y}=R
\end{aligned}
$$

Where $K>1$ is a constant that spreads out-the-screen items.

This function allows us to move all items correctly, and by defining a number of visible objects we can adapt the visualization to the device.

\subsection{Step-by-step navigation}

One major issue raised by HCI researchers is the importance of animations or animated transitions for user's understanding of interface behavior [24] or decision-making [25]. These animations should therefore be cancellable if user stops interacting, allowing him to undo his action in a fluid way [26]. That is why we modeled a Behavioral State Machine for each of our widget's items which describes items states and transitions (Figure 12).

On the Figure 12, one can notice that Anim states represent animated transitions from one circle to another, allowing the cancellation of actions and the understanding of interaction. All items are initially on the Init state, and depending if they are at the first level of hierarchy, they go either on the first circle or hidden below their fathers. In Unity3D, we put children at the same position of fathers, but we deactivate their sprite renderer and box collider, disabling their interactive capabilities. $\mathbf{u C 1}, \mathbf{s C 1}, \mathbf{u C 2}$ and $\mathbf{s C 2}$ corresponds respectively to unselected state on circle one, selected state on circle one and the same for circle two. C0 and C3 Hidden states correspond to specific hidden states which represents non-interactive states displayed respectively at the origin of the widget and above the second circle, as represented on Figure 7.

When a user selects an item on the first circle for the first time (Double arrow), this item goes from $\mathbf{u C 1}$ to $\mathbf{s C 1}$, and its children goes from Hidden to 


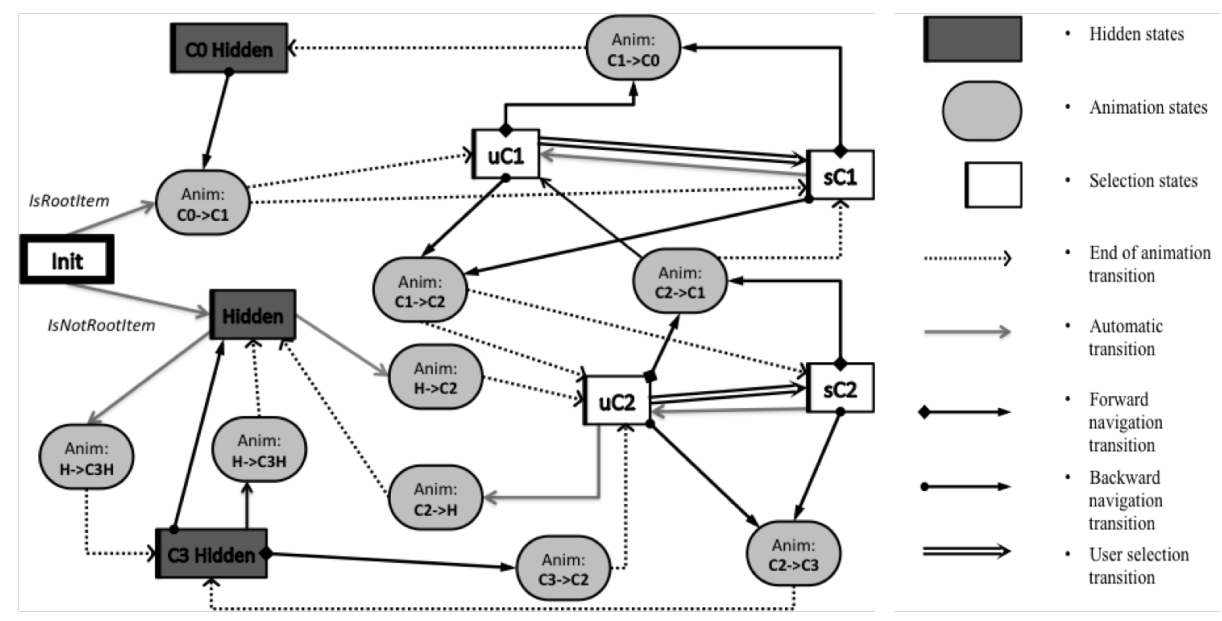

Fig. 12. Item's Behavior

uC2. If user selects another item from the first circle, formerly selected item goes from $\mathbf{s C 1}$ to $\mathbf{u C 1}$, its children from $\mathbf{u C 2}$ to Hidden and the newly selected item and its children behaves as previously described. When a user selects an item on the second circle, it goes from $\mathbf{u C 2}$ to $\mathbf{s C 2}$ and its children goes from Hidden to C3 Hidden. Selecting another item from second circle acts as previously said for circle one. When a user perform a forward navigation (square-beginning arrows), items from circle one disappeared and goes to $\mathbf{C 0}$, origin of the widget, but we are saving their status (selected, unselected) for backward navigation. Items from second circles goes on the first one, according to their selected statuses, and items from third hidden circle goes all unselected on the second one.

Backward navigation (round-beginning arrows) makes items hidden in circle zero to move to circle one, items in circle one to circle two with same statuses and items from circle three to Hidden state, under their parent.

We also add a global state machine for the widget, for controlling the global behavior and saving statuses of items (if an item is selected, its siblings should be deselected and so on).

This section described the implementation and development of RICHIE, a step-by-step hierarchy exploration widget. The following one will present shortly the Command and Control application in which we implemented and tested it, and other domains we think it can be used for. We will describe also our on-going efforts to evaluate usability of our system.

\section{Tests and Perspectives}

C2 systems usually deal with large and imbalanced hierarchies of units displayed within a map and in a linear classic way [27]. These constraints limits the screen 
space used by the hierarchy visualization and its interaction, that is why existing multitouch C2 systems were developed usually for interactive tabletops [28, 29]. As we want to adapt an existing C2 system showed in Figure 7 to handheld tactile devices, we are currently testing a C2-tactile prototype which embeds our RICHIE widget (Figure 13). Our widget might be used in every domain that

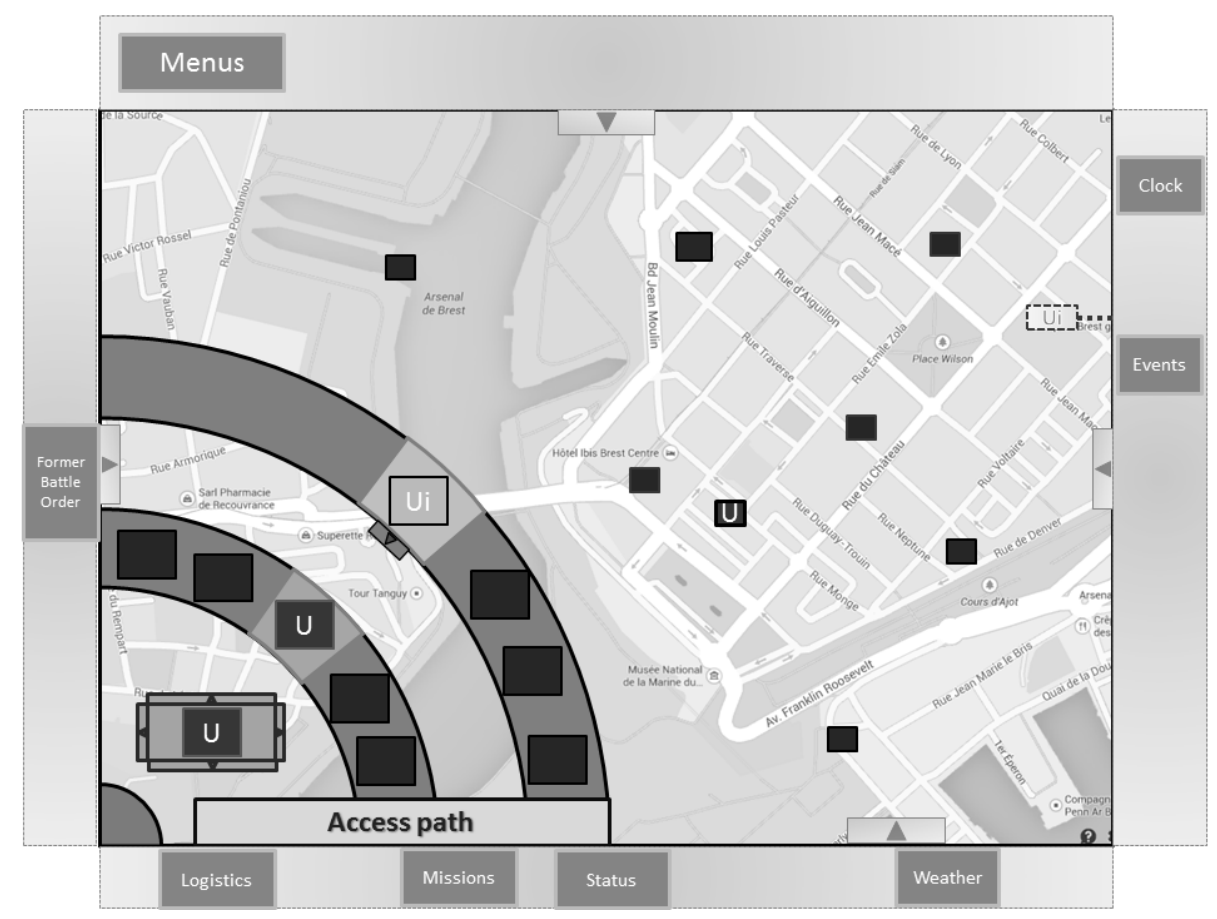

Fig. 13. Sketch of our adaptation.

need browsing through large hierarchies without displaying the whole dataset. Genomic data visualization systems such as those proposed by Shaer et al. [30] could, for example, benefit from our solution. Semantic relationships between elements could also be explored, such as with the MASCARET Virtual Environment meta-model [31]; our widget could facilitate the exploration of information towards a simulation.

We will perform experimentations in order to test different ways of interaction (for example adding a breadcrumb trail will allow users to jump from one level of hierarchy to a previous one, as in file systems or websites [32,33]). Simultaneous map interaction and hierarchy exploration will be evaluated too [34]. Performance comparisons between RICHIE and existing exploration techniques for item retrieval or other specific tasks would bring cues of user's preferences $[35]$. 


\section{Conclusion}

This paper has presented RICHIE, a step-by-step widget that allows the exploration through larges data hierarchies on tactile handheld devices. Unlike other approaches, it uses minimal on-screen space, enabling the interaction with distinct tools, such as interactive maps. Users do not dispose of a global view of data, but instead they navigate level after level through hierarchy. Our immediate future work is to made experimentations in order to explore RICHIE's capabilities and usages in different applications and on different devices.

\section{Acknowledgments}

This research work was partially funded by the "Ministère de l'Enseignement Supérieur et de la Recherche", the "Conseil Général du Finistère", the "Brest Métropole Océane", and especially the "Agence Nationale de la Recherche" (TACTIC ANR Project, ANR-12-ASTR-0020).

\section{References}

1. I. Herman, G. Melançon, and M. S. Marshall, "Graph visualization and navigation in information visualization: A survey," IEEE Transactions on Visualization and Computer Graphics, vol. 6, pp. 24-43, Jan. 2000.

2. G. Draper, Y. Livnat, and R. Riesenfeld, "A survey of radial methods for information visualization," Visualization and Computer Graphics, IEEE Transactions on, vol. 15, pp. 759-776, Sept 2009.

3. C. Appert, J.-D. Fekete, et al., "Controltree: Navigating and selecting in a large tree," in ACM symposium on User interface software and technology, 2006.

4. J. Hao, C. A. Gabrysch, C. Zhao, Q. Zhu, and K. Zhang, "Visualizing and navigating hierarchical information on mobile user interfaces," International Journal of Advanced Intelligence, vol. 2, no. 1, pp. 81-103, 2010.

5. J. Yang, M. O. Ward, and E. A. Rundensteiner, "Interring: An interactive tool for visually navigating and manipulating hierarchical structures," in Proceedings of the IEEE Symposium on Information Visualization (InfoVis'02), INFOVIS '02, (Washington, DC, USA), pp. 77-84, IEEE Computer Society, 2002.

6. C. McCann and R. Pigeau, "Clarifying the concepts of control and of command," in Proceedings of the 1999 Command and Control Research and Technology Symposium, vol. 29, 1999.

7. A. P. Chhetri, K. Zhang, and E. Jain, "Erelt: A faster alternative to the list-based interfaces for tree exploration and searching in mobile devices," in Proceedings of the 6th International Symposium on Visual Information Communication and Interaction, VINCI '13, (New York, NY, USA), pp. 54-63, ACM, 2013.

8. N. Sheth, K. Borner, J. Baumgartner, K. Mane, and E. Wernert, "Treemap, radial tree, and 3d tree visualizations," IEEE InfoVis Poster Compendium, vol. 1, pp. 128-129, 2003. 
9. S. Schmidt, M. A. Nacenta, R. Dachselt, and S. Carpendale, "A set of multi-touch graph interaction techniques," in ACM International Conference on Interactive Tabletops and Surfaces, ITS '10, (New York, NY, USA), pp. 113-116, ACM, 2010.

10. A. Holzinger, B. Ofner, and M. Dehmer, "Multi-touch graph-based interaction for knowledge discovery on mobile devices: State-of-the-art and future challenges," in Interactive Knowledge Discovery and Data Mining in Biomedical Informatics (A. Holzinger and I. Jurisica, eds.), vol. 8401 of Lecture Notes in Computer Science, pp. 241-254, Springer Berlin Heidelberg, 2014.

11. C. Plaisant, J. Grosjean, and B. B. Bederson, "Spacetree: Supporting exploration in large node link tree, design evolution and empirical evaluation," in Proceedings of the IEEE Symposium on Information Visualization (InfoVis'02), INFOVIS '02, (Washington, DC, USA), pp. 57-, IEEE Computer Society, 2002.

12. Q. V. Nguyen and M. L. Huang, "Enccon: an approach to constructing interactive visualization of large hierarchical data," Information Visualization, vol. 4, no. 1, pp. 1-21, 2005.

13. B. Johnson and B. Shneiderman, "Tree-maps: A space-filling approach to the visualization of hierarchical information structures," in Proceedings of the 2Nd Conference on Visualization '91, VIS '91, (Los Alamitos, CA, USA), pp. 284-291, IEEE Computer Society Press, 1991.

14. K. Seipp and K. Devlin, "The one hand wonder: A framework for enhancing onehanded website operation on touchscreen smartphones," in Proceedings of the 10th International Conference on Web Information Systems and Technologies, WEBIST'14, (Barcelona), Goldsmiths Research Online, 2014.

15. J. Wagner, S. Huot, and W. Mackay, "Bitouch and bipad: Designing bimanual interaction for hand-held tablets," in Proceedings of the SIGCHI Conference on Human Factors in Computing Systems, CHI '12, (New York, NY, USA), pp. 23172326, ACM, 2012.

16. K. Wolf and N. Henze, "Comparing pointing techniques for grasping hands on tablets," in Proceedings of the 16th International Conference on Human-computer Interaction with Mobile Devices 6 \#38; Services, MobileHCI '14, (New York, NY, USA), pp. 53-62, ACM, 2014.

17. S. Huot and E. Lecolinet, "Focus+context visualization techniques for displaying large lists with multiple points of interest on small tactile screens," in Proceedings of the 11th IFIP TC 13 International Conference on Human-computer Interaction - Volume Part II, INTERACT'07, (Berlin, Heidelberg), pp. 219-233, SpringerVerlag, 2007.

18. Y. S. Park, S. H. Han, J. Park, and Y. Cho, "Touch key design for target selection on a mobile phone," in Proceedings of the 10th International Conference on Human Computer Interaction with Mobile Devices and Services, MobileHCI '08, (New York, NY, USA), pp. 423-426, ACM, 2008.

19. T. J. Jankun-Kelly and K.-L. Ma, "Moiregraphs: Radial focus+context visualization and interaction for graphs with visual nodes," in Proceedings of the Ninth Annual IEEE Conference on Information Visualization, INFOVIS'03, (Washington, DC, USA), pp. 59-66, IEEE Computer Society, 2003.

20. T. Hesselmann, S. Flöring, and M. Schmitt, "Stacked half-pie menus: Navigating nested menus on interactive tabletops," in Proceedings of the ACM International Conference on Interactive Tabletops and Surfaces, ITS '09, (New York, NY, USA), pp. 173-180, ACM, 2009.

21. J. Francone, G. Bailly, E. Lecolinet, N. Mandran, and L. Nigay, "Wavelet menus on handheld devices: Stacking metaphor for novice mode and eyes-free selection for 
expert mode," in Proceedings of the International Conference on Advanced Visual Interfaces, AVI '10, (New York, NY, USA), pp. 173-180, ACM, 2010.

22. K. Kin, M. Agrawala, and T. DeRose, "Determining the benefits of direct-touch, bimanual, and multifinger input on a multitouch workstation," in Proceedings of Graphics Interface 2009, GI '09, (Toronto, Ont., Canada, Canada), pp. 119-124, Canadian Information Processing Society, 2009.

23. A. Cockburn, D. Ahlström, and C. Gutwin, "Understanding performance in touch selections: Tap, drag and radial pointing drag with finger, stylus and mouse," Int. J. Hum.-Comput. Stud., vol. 70, pp. 218-233, Mar. 2012.

24. B.-W. Chang and D. Ungar, "Animation: From cartoons to the user interface," in Proceedings of the 6th Annual ACM Symposium on User Interface Software and Technology, UIST '93, (New York, NY, USA), pp. 45-55, ACM, 1993.

25. C. Gonzalez, "Does animation in user interfaces improve decision making?," in Proceedings of the SIGCHI Conference on Human Factors in Computing Systems, CHI '96, (New York, NY, USA), pp. 27-34, ACM, 1996.

26. N. Elmqvist, A. Vande Moere, H.-C. Jetter, D. Cernea, H. Reiterer, and T. J. Jankun-Kelly, "Fluid interaction for information visualization," Information Visualization, vol. 10, pp. 327-340, Oct. 2011.

27. L. Ucuzal and A. Kopar, "Gis (geographic information systems) in ccis (command \& control systems)," Geographic Information, Conversion and Management Systems, vol. 30, p. 6, 2010.

28. C. Bortolaso, M. Oskamp, T. N. Graham, and D. Brown, "Ormis: A tabletop interface for simulation-based training," in Proceedings of the 2013 ACM International Conference on Interactive Tabletops and Surfaces, ITS '13, (New York, NY, USA), pp. 145-154, ACM, 2013.

29. R. Szymanski, M. Goldin, N. Palmer, R. Beckinger, J. Gilday, and T. Chase, "Command and control in a multitouch environment." 26th Army Science Conference, 2008.

30. O. Shaer, M. Strait, C. Valdes, H. Wang, T. Feng, M. Lintz, M. Ferreirae, C. Grote, K. Tempel, and S. Liu, "The design, development, and deployment of a tabletop interface for collaborative exploration of genomic data," Int. J. Hum.-Comput. Stud., vol. 70, pp. 746-764, Oct. 2012.

31. C. Buche, R. Querrec, P. De Loor, and P. Chevaillier, "Mascaret: pedagogical multi-agents systems for virtual environment for training," in Cyberworlds, 2003. Proceedings. 2003 International Conference on, pp. 423-430, Dec 2003.

32. W. Hudson, "Breadcrumb navigation: There's more to hansel and gretel than meets the eye," interactions, vol. 11, pp. 79-80, Sept. 2004.

33. A. Smith, T. Hawes, and M. Myers, "Hiérarchie: Interactive visualization for hierarchical topic models," in Proceedings of the Workshop on Interactive Language Learning, Visualization, and Interfaces, pp. 71-78, Association for Computational Linguistics, 2014.

34. E. Pietriga, C. Appert, and M. Beaudouin-Lafon, "Pointing and beyond: An operationalization and preliminary evaluation of multi-scale searching," in Proceedings of the SIGCHI Conference on Human Factors in Computing Systems, CHI '07, (New York, NY, USA), pp. 1215-1224, ACM, 2007.

35. J. Stasko, "An evaluation of space-filling information visualizations for depicting hierarchical structures," Int. J. Hum.-Comput. Stud., vol. 53, pp. 663-694, Nov. 2000 . 